\title{
A Monoclonal Antibody to the Carboxyterminal Domain of Procollagen Type I Visualizes Collagen-synthesizing Fibroblasts

\author{
Detection of an Altered Fibroblast Phenotype in Lungs of Patients with Pulmonary Fibrosis
}

John A. McDonald," Thomas J. Broekelmann," Mary Lou Matheke," Edmond Crouch,‡ Michele Koo,§ and Charles Kuhn III\| *Pulmonary Disease Division, Departments of Medicine and Biochemistry, $\ddagger$ Departments of Pathology, The Jewish Hospital of St. Louis, §Plastic Surgery and \|Department of Pathology, Washington University School of Medicine, St. Louis, Missouri 63110

\begin{abstract}
Excessive collagen deposition plays a critical role in the development of fibrosis, and early or active fibrosis may be more susceptible to therapeutic intervention than later stages of scarring. However, at present there is no simple method for assessing the collagen-synthesizing and secreting activity of fibroblasts in human tissues. Type I procollagen carboxyterminal domains are proteolytically removed during collagen secretion. Thus, antibodies to these domains should stain fibroblasts synthesizing type I collagen but not extracellular collagen fibrils which could mask the signal from the cells. We developed and characterized a monoclonal antibody (Anti-pC) specific for the carboxyterminal propeptide of type I procollagen. To determine the relationship between Anti-pC staining and collagen synthesis, we stained embryonic and adult chicken tendon. Embryonic chick tendon fibroblasts actively synthesizing type I collagen stained heavily with Anti-pC, while quiescent adult tendon fibroblasts did not stain with Anti-pC. Wounded adult tendons developed fibroblasts that stained with Anti-pC at the wound site. Thus, Anti-pC specifically visualized fibroblasts actively synthesizing collagen. Lung biopsies from patients with fibrotic lung disease were stained with Anti-pC. Interstitial and intraalveolar fibroblasts in biopsies from patients with active fibrosis stained intensely with Anti-pC, while normal human lung was unstained. The absence of staining in normal lung supports the hypothesis that fibrosis is associated with an altered collagen-synthesizing phenotype of tissue fibroblasts. Anti-pC may provide a useful clinical tool for assessing fibrogenic activity at sites of tissue injury.
\end{abstract}

\section{Introduction}

The fibrotic lung diseases are a heterogeneous group of disorders reflecting a common pathophysiologic response of the lung to injury. Whereas in many cases the etiology is clear, cases are frequently of unknown cause, thus creating a significant therapeutic dilemma. Although the pathophysiologic mechanisms remain unclear, progressive connective tissue deposition characterizes fibrotic lung disorders (1). Histopathological and clinical findings currently guide therapy (2) but there is a clear need for

Address reprint requests to John A. McDonald, Ph.D., M.D., Pulmonary Disease Division, Washington University School of Medicine, Box 8052, 660 South Euclid, St. Louis, MO 63110. 1986.

Received for publication 28 March 1986 and in revised form 30 June

J. Clin. Invest.

(C) The American Society for Clinical Investigation, Inc.

0021-9738/86/11/1237/08 $\$ 1.00$

Volume 78, November 1986, 1237-1244 more objective methods to assess disease activity. Determining collagen content of lung biopsies is not a reliable guide to disease activity. In addition to the obvious methodological difficulties, lung collagen may accumulate slowly or explosively, or even exist in excessive quantities as a result of prior disease activity $(1,3,4)$. Furthermore, chemically detectable increases in collagen content may occurr relatively late and thus may not be detectable as a result of focal fibroproliferative reactions. On the other hand, detecting the presence of cells actively secreting collagen in the lung would suggest active connective tissue deposition, i.e., fibrosis. Accordingly, some measure of the numbers and collagensecreting activities of fibroblasts in fibrotic lung could be useful to guide therapy. However, no simple tool to assess the collagensecreting activity of lung fibroblasts in biopsy specimens has emerged to date.

Because type I collagen propeptides are rapidly removed by proteolysis after collagen secretion (5), antibodies to these peptides might detect cells actively synthesizing collagen by yielding specific intracellular staining without the problems of background staining of preexisting extracellular collagen. Such antibodies might provide results similar to in situ hybridization of collagen mRNA with a cDNA. To test this hypothesis, we developed and characterized a monoclonal antibody to the carboxy terminal domain of human type I procollagen (Anti-pC) ${ }^{1}$ and validated its use in a model system of collagen synthesis, embryonic and adult chicken tendon. In the chicken model, Anti-pC yielded selective intracellular staining of fibroblastic cells in embryonic tendon fibroblasts actively synthesizing type I procollagen (6). Adult tendons, where collagen synthesis was minimal, were not stained. However, after laceration and repair, abundant fibroblasts staining for intracellular procollagen appeared at the wound site. We then used Anti-pC to stain normal and fibrotic human lung. Healthy adult human lung did not stain with Anti-pC, whereas fibrotic lung from patients with clinically active pulmonary fibrosis contained abundant staining fibroblasts. These results suggest that Anti-pC selectively detects fibroblasts synthesizing type I procollagen and therefore represents a useful tool for the study of fibroproliferative disorders and wound healing in general.

\section{Methods}

Monoclonal antibody production. Procollagen type I was purified from ascorbate-supplemented human fetal lung fibroblast (IMR-90) cultures by ammonium sulfate precipitation and DEAE-cellulose chromatography of spent medium (7). BALB/c mice were immunized with intact type I

1. Abbreviations used in this paper: Anti-pC, monoclonal IgG to carboxyterminal domain of type I procollagen; ELISA, enzyme-linked immunosorbent assay; IMR-90, human fetal lung fibroblast; TBS, $7.5 \mathrm{mM}$ Tris- $\mathrm{HCl}-150 \mathrm{mM} \mathrm{NaCl}$, pH 7.3, containing $1 \%$ bovine serum albumin. 


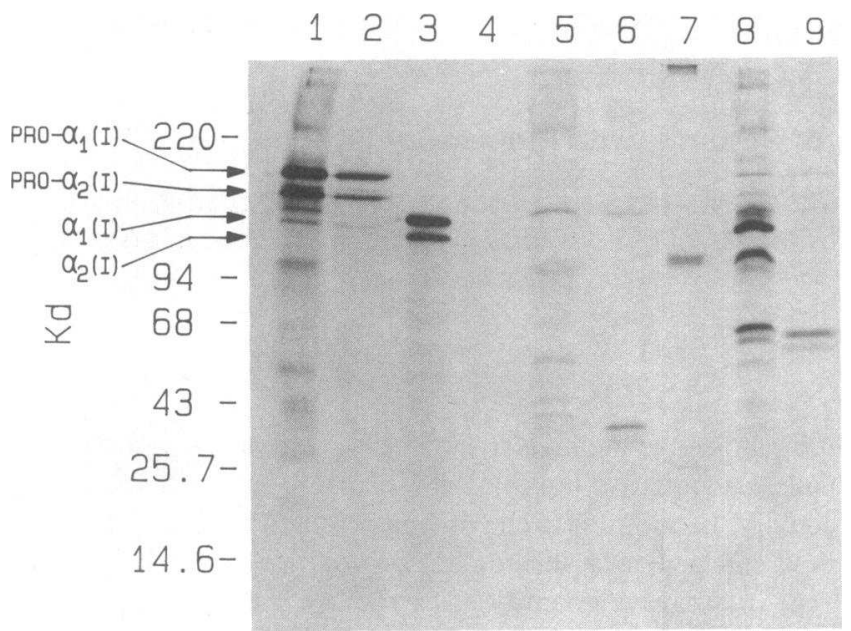

Figure 1. Immunoprecipitation of pepsin and collagenase digests of $\left[{ }^{14} \mathrm{C}\right]$ proline-labeled IMR-90 fibroblast medium with Anti-pC. This is a fluorogram of a 7.5\% SDS-PAGE gel electrophoresis run reduced with dithiothreitol (lane 7 is unreduced). The relative mass of the standards is shown at left in kilodaltons (220, fibronectin monomer; 94, phosphorylase b; 68 , bovine serum albumin; 43 , ovalbumin; 25.7 , chymotrypsinogen; 14.6, lysozyme). The positions of procollagen and collagen $\alpha$-chains are indicated. Lanes 1-4 are from the pepsin digestion experiment. Lane $1,\left[{ }^{14} \mathrm{C}\right]$ proline-labeled medium proteins from IMR-90 after removal of gelatin-binding polypeptides. Lane 2, sample displayed in lane 1 immunoprecipitated with Anti-pC. Lane 3, sample in lane 1 digested with pepsin. Lane 4, Anti-pC immunoprecipitate of the sample in lane 3 . Note that pepsin digestion converts the procollagen chains to collagen $\alpha$-chains (lane 3), and that Anti-pC does not immunoprecipitate the pepsinized collagen (lane 4). Lanes 5-7 are from the bacterial collagenase digestion experiment. Lane 5, digest of unfractionated fibroblast medium shown in lane 1 . The procollagen bands disappear, but other noncollagenous polypeptides seen in lane 1 remain. Lane 6, immunoprecipitate of the sample in lane 5 containing $35-$ and $32-\mathrm{kD}$ polypeptides (reduced). The lower polypeptide is not easily seen in this figure. Lane 7, immunoprecipitate run unreduced (this lane is from a parallel gel). The polypeptide immunoprecipitated by Anti-pC runs with a molecular mass of $110 \mathrm{kD}$ unreduced. Lanes 8 and 9 show digests of labeled fibroblast medium displayed in lane 1 with purified human skin collagenase. The total digest is displayed in lane 8; the immunoprecipitated sample is in lane 9. The total digest contains pairs of labeled polypeptides of 120 and $105 \mathrm{kD}$ representing the pro-TCA fragments and 56 and $63 \mathrm{kD}$ representing the pro-TCB fragments resulting from mammalian collagenase cleavage of type I procollagen (13). Anti-pC immunoprecipitated the pro-TCB fragments (lane 9).

procollagen, spleen cells fused with Sp2/0-Ag14 myeloma cells, and growth-positive clones screened for type I procollagen reactivity by enzyme-linked immunosorbent assay (ELISA). The protocols used for monoclonal antibody production with $\mathrm{Sp} 2 / 0-\mathrm{Ag} 14$ myeloma cells including immunization, cloning, and ELISA assays have been published previously (8-10). Anti-pC, which stained IMR-90 matrix and immunoprecipitated procollagen type I from labeled fibroblast medium, was further characterized.

Metabolic labeling and immunoprecipitation. Metabolic labeling of IMR-90 fibroblasts with $\left[{ }^{35} \mathrm{~S}\right]$ cysteine and methionine or $\left[{ }^{14} \mathrm{C}\right]$ proline and immunoprecipitation of IMR-90 medium with Anti-pC were performed as previously described (9). In some experiments, fibronectin was removed from labeled medium by gelatin-Sepharose absorption before immunoprecipitation to prevent its nonspecific precipitation.

Immunological reagents. Polyclonal rabbit anti-human plasma fibronectin and preimmune rabbit IgG were purified as described (11).
Monoclonal mouse IgG was purified from ascites by precipitation with ammonium sulfate, molecular sieve chromatography on Sephacryl S200 (Pharmacia, Inc., Piscataway, NJ), and DEAE chromatography. Ascites fluid (20-25 ml) containing protease inhibitors ( $1 \mathrm{mM}$ phenylmethylsulfonylfluoride, $1 \mathrm{mM} N$-ethylmaleimide, $20 \mathrm{mM}$ EDTA, and $20 \mathrm{mM}$ $\epsilon$-amino caproic acid in $50 \mathrm{mM}$ Tris- $\mathrm{HCl}, \mathrm{pH} 7.4$ at $25^{\circ} \mathrm{C}$ was clarified by centrifugation $(20,000 \mathrm{~g}, 30 \mathrm{~min})$. An equal volume of $50 \mathrm{mM}$ sodium borate containing $200 \mathrm{mM} \mathrm{NaCl}, \mathrm{pH} 7.5$, was added to the supernate followed by solid ammonium sulfate $(29 \mathrm{~g} / 100 \mathrm{ml} ; 50 \%$ saturation at $0^{\circ} \mathrm{C}$ ). After $4 \mathrm{~h}$ on ice, the precipitate containing IgG was collected by centrifugation and dissolved in $20 \mathrm{ml}$ of $7.5 \mathrm{mM}$ Tris-HCl-150 mM $\mathrm{NaCl}, \mathrm{pH}$ 7.4. After centrifugation, the sample was applied to a $5 \times 120$ cm column of Sephacryl S-200 equilibrated with $50 \mathrm{mM}$ Tris-HCl, $\mathrm{pH}$ 7.4. The peak containing IgG was then applied to a $2.5 \times 10 \mathrm{~cm}$ column of DEAE-Sephacryl (Pharmacia, Inc.). The column was washed with 50 $\mathrm{mM}$ Tris- $\mathrm{HCl}, \mathrm{pH} 7.4$, and eluted with a linear gradient of $\mathrm{NaCl}$ from 0 to $0.5 \mathrm{M}$ in the same buffer. Anti-pC eluted at $\sim 80 \mathrm{mM} \mathrm{NaCl}$ with a typical recovery of $100 \mathrm{mg}$ IgG from 20-25 ml of ascites. SDS-polyacrylamide gel electrophoresis (12), Coomassie Blue staining, and quantitative laser scanning densitometry showed that all monoclonal and polyclonal Ig preparations used in this study were at least $90-95 \%$ pure.

Mapping of the Anti-pC binding domain using procollagen digestion products. Procollagen was subjected to digestion with pepsin, skin collagenase, and bacterial collagenase according to published methods $(9$, 13-15). Crystalline pepsin (Sigma P 6887) and highly purified bacterial collagenase (type VII; see reference 9) were purchased from Sigma Chemical Co., St. Louis, MO.

Immunohistochemistry. Double label immunofluorescence staining of unpermeabilized fibroblasts for fibronectin and procollagen was performed as described (16) using rhodamine isothiocyanate-conjugated, affinity-purified rabbit anti-human plasma fibronectin IgG and Anti$\mathrm{pC}$, followed by fluorescein isothiocyanate-conjugated mouse-specific goat anti-mouse IgG. Control human lung was obtained from uninvolved areas of three lobectomy specimens performed for localized peripheral lung carcinomas. Samples of interstitial disease came from four open biopsies and two autopsies, as indicated in Table I. Immunofluorescence staining of lung was carried out on fresh frozen sections.

For immunoperoxidase staining, acetic acid-ethanol fixative (17) was injected through the pleura into the lung specimen to inflate the collapsed lung. The lung was immersed in the fixative overnight and embedded in paraffin wax. Sections were collected on gelatin-coated slides and air dried. Deparaffinized slides were rehydrated in $7.5 \mathrm{mM}$ Tris-HCl-150 $\mathrm{mM} \mathrm{NaCl}, \mathrm{pH} \mathrm{7.3,} \mathrm{containing} \mathrm{1 \%} \mathrm{bovine} \mathrm{serum} \mathrm{albumin} \mathrm{(TBS)} \mathrm{for} 20$

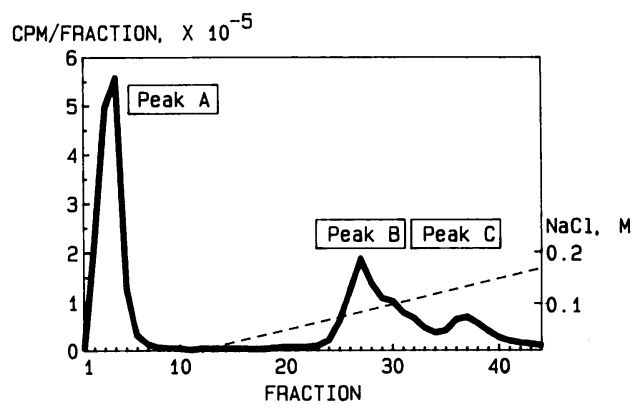

Figure 2. Isolation of type I procollagen and partially processed collagens from fibroblast medium by DEAE-cellulose chromatography. ${ }^{35}$ S]Methionine and ${ }^{35}$ S $]$ cysteine-labeled IMR-90 fibroblast medium was precipitated with ammonium sulfate and chromatographed on a DE-52 DEAE-cellulose column in $50 \mathrm{mM}$ Tris- $\mathrm{HCl}, 2 \mathrm{M}$ urea, $\mathrm{pH}$ 8.3. After elution of the nonbound material in peak $A$, the column was eluted with a 0 to $0.2 \mathrm{M} \mathrm{NaCl}$ gradient (dashed line). Peak $A$ contains type I pC-collagen, fully processed collagen, and small amounts of pN-collagen. Peak $B$ contains intact type I procollagen and pC-collagen (see Fig. 3). Peak $C$ contains type III procollagen, which was not immunoprecipitated with Anti-pC (not shown). 
Table I. Patient Characteristics

\begin{tabular}{|c|c|c|c|c|}
\hline Patient & Diagnosis & Procollagen staining & Clinical course & Histological diagnosis \\
\hline \multicolumn{5}{|l|}{ Controls } \\
\hline A.S. & Lung cancer & Negative & Solitary nodule & Normal lung \\
\hline E.A. & Lung cancer & Negative & Solitary nodule & Normal lung \\
\hline T.L.B. & Lung cancer & Negative & Solitary nodule & Normal lung \\
\hline \multicolumn{5}{|c|}{ Pulmonary fibrosis } \\
\hline L.N. & $\begin{array}{l}\text { Leucocytoclastic angiitis, } \\
\text { renal and respiratory } \\
\text { failure }\end{array}$ & $\begin{array}{l}\text { Marked intraalveolar staining for } \\
\text { fibronectin and procollagen }\end{array}$ & $\begin{array}{l}\text { Pulmonary fibrosis after } \\
\text { acute respiratory failure }\end{array}$ & $\begin{array}{l}\text { Diffuse alveolar damage, } \\
\text { bronchiolitis obliterans }\end{array}$ \\
\hline D.G. & Idiopathic pulmonary fibrosis & Focal groups of positive fibroblasts & Rapidly progressive course & Severe fibrosis and remodeling \\
\hline E.M. & Idiopathic pulmonary fibrosis & Very few positive cells & $\begin{array}{l}\text { 10-yr history slowly } \\
\text { progressive }\end{array}$ & Severe fibrosis and remodeling \\
\hline C.S. & $\begin{array}{c}\text { Rheumatoid arthritis, } \\
\text { pulmonary fibrosis }\end{array}$ & $\begin{array}{l}\text { Many positive interstitial } \\
\text { fibroblasts }\end{array}$ & $\begin{array}{l}\text { Rapidly progressive } \\
\text { disease }\end{array}$ & Severe fibrosis and remodeling \\
\hline M.C. & Eosinophilic granuloma & $\begin{array}{l}\text { Few positive fibroblasts in focal } \\
\text { areas of scarring }\end{array}$ & $\begin{array}{l}\text { Indolent disease (no } \\
\text { progression in } 6 \mathrm{mo} \text { ) }\end{array}$ & Eosinophilic granuloma \\
\hline W.J. & Eosinophilic granuloma & $\begin{array}{l}\text { Few positive fibroblasts in focal } \\
\text { areas of scarring }\end{array}$ & Indolent disease & Eosinophilic granuloma \\
\hline
\end{tabular}

min and reacted for 30 or 60 min with Anti-pC $(25 \mu \mathrm{g} / \mathrm{ml})$ or normal mouse serum. After rinsing in TBS, bound mouse Ig was visualized by the avidin-biotin complex method with a commercial kit (Vectastain $\mathrm{ABC}$ Kit, Vector Laboratories, Burlingame, $\mathrm{CA}$ ) in accordance with the manufacturer's recommendations. In some cases, the sections were counterstained for $1 \mathrm{~min}$ with hematoxylin to reveal cellular detail.

Patient population. Patients referred to the Pulmonary Disease Divisions at the Washington University Medical Center, St. Louis, MO, for evaluation of unexplained dyspnea and abnormal chest roentgenograms underwent open lung biopsy for diagnostic purposes. Evidence for clinical progression included one or more of the following signs or symptoms (2): (a) increasing dyspnea with no other discernable cause; (b) deteriorating pulmonary function tests over the previous 3-6 mo; (c) increasing roentgenographic abnormalities on serial chest $\mathrm{x}$-ray; $(d)$ acute respiratory failure with no previous history of respiratory disease and a fatal outcome. Patients were judged to have quiescent disease when there was no change in categories $1-3$ for a minimum of 6 mo after the biopsy.

Animal models. Anti-pC recognized both chicken and calf procollagen type I, so we used the chicken flexor tendon as a model tissue synthesizing type I collagen to examine the relationship between staining of fibroblasts with Anti-pC and collagen synthesis. Immunohistochemical studies were performed on 16-d chick embryos and adult white male leghorn chickens. Intact embryos and excised adult tendons were fixed in acetic aid-ethanol fixative and embedded, and the paraffin sections processed identically to those of lung specimens. For studies of tendon healing, we used the animal model of Birdsell et al. (18), who demonstrated more than 10fold increases in collagen synthesis at the wound site of lacerated and repaired chicken flexor digitorum profundus tendons, and much smaller increases in collagen synthesis in the same tendon distal to the wound site (18). The tendon was exposed, lacerated, sutured, and replaced in

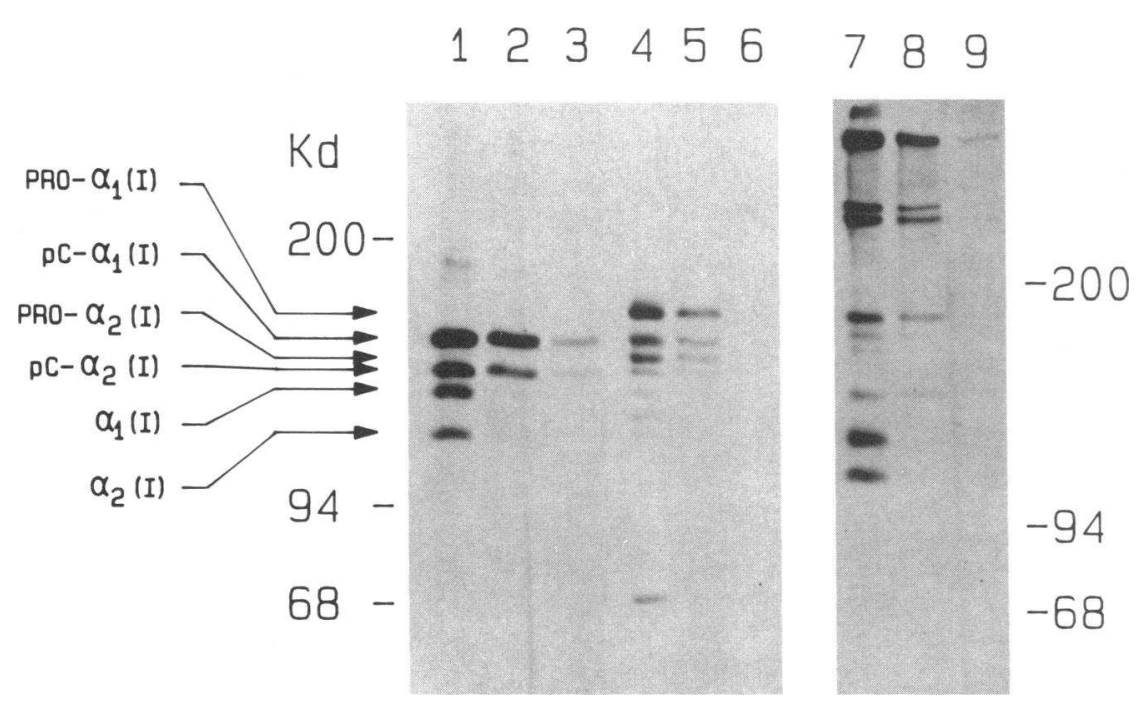

Figure 3. Immunoprecipitation of partially processed type I procollagen with Anti-pC. This is a fluorogram of samples run on a 5\% SDSPAGE gel. Lanes 1-6 (left) are run reduced, and 7-9 (right) are run unreduced. Molecular mass standards are as shown in Fig. 1. Lanes 1-3 are from peak $A$ of Fig. 2 before and after immunoprecipitation with Anti-pC or preimmune IgG. Lane 1 displays the total labeled polypeptides, lane 2 is an Anti-pC immunoprecipitate of the sample shown in lane 1 , and lane 3 is a preimmune IgG control. Anti-pC immunoprecipitates only the upper pair of disulfide-bonded collagenous polypeptides, and not the nondisulfide crosslinked type I collagen chains. The preimmune control in lane 3 contains only traces of pC-collage $\alpha$-chains. Lanes 4-6 are peak $B$ from Fig. 2 run alone (lane 4), immunoprecipitated with Anti-pC (lane 5), or preimmune IgG (lane 6). Note that Anti-pC immunoprecipitated both type I procollagen and pC-collagen $\alpha$-chains. Lanes 7-9 are the

unreduced samples corresponding to lanes 1-3. Note that the polypeptides immunoprecipitated with Anti-pC are all disulfide crosslinked, the major polypeptide migrating near the top of the resolving gel as expected for type I procollagen. The lower weight polypeptides are compatible with type I procollagen processing intermediates (see references 5 and 13). 

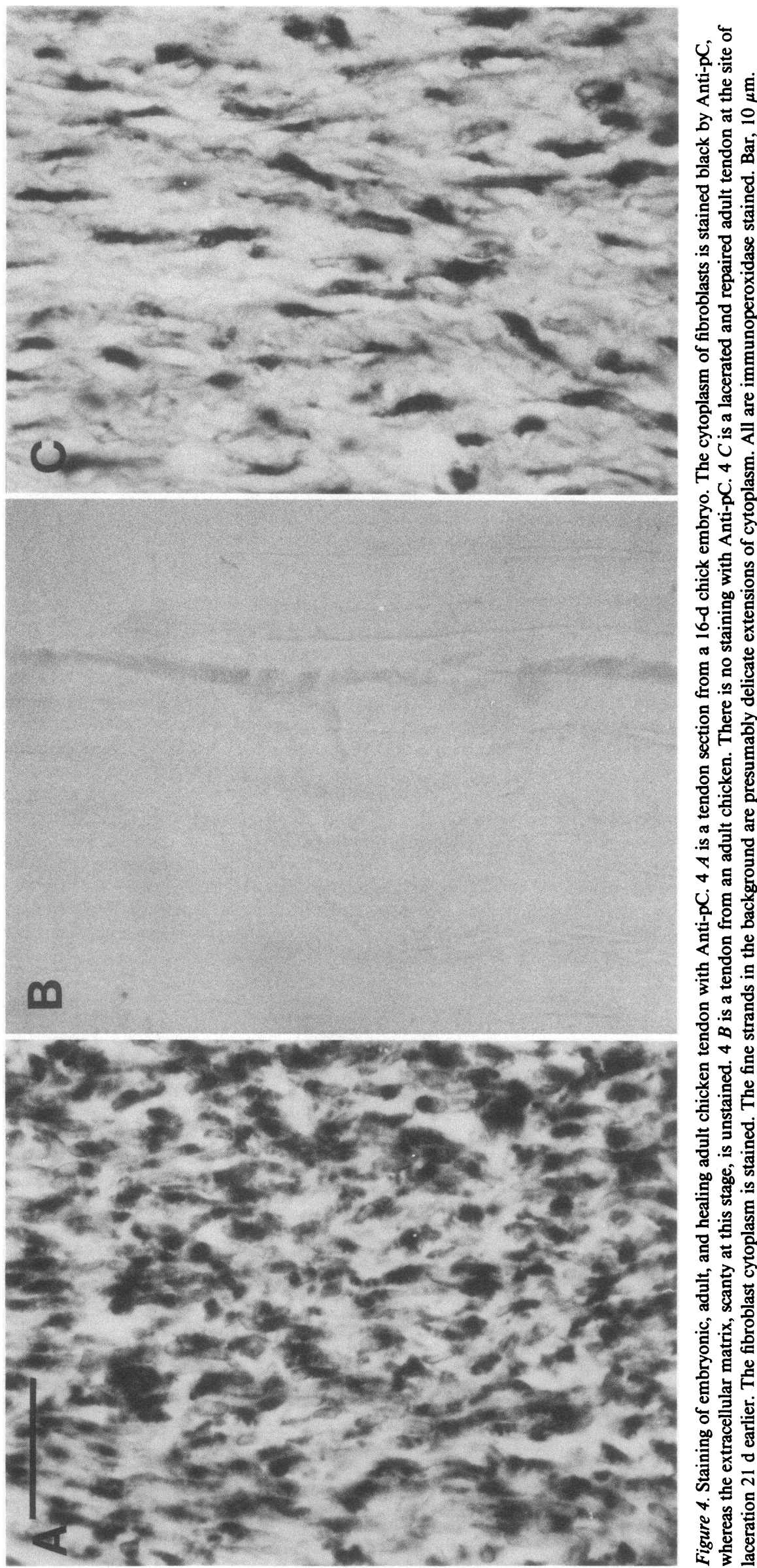

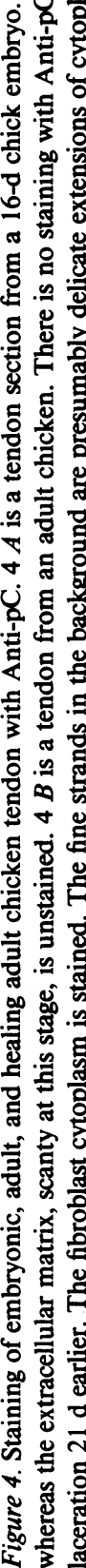




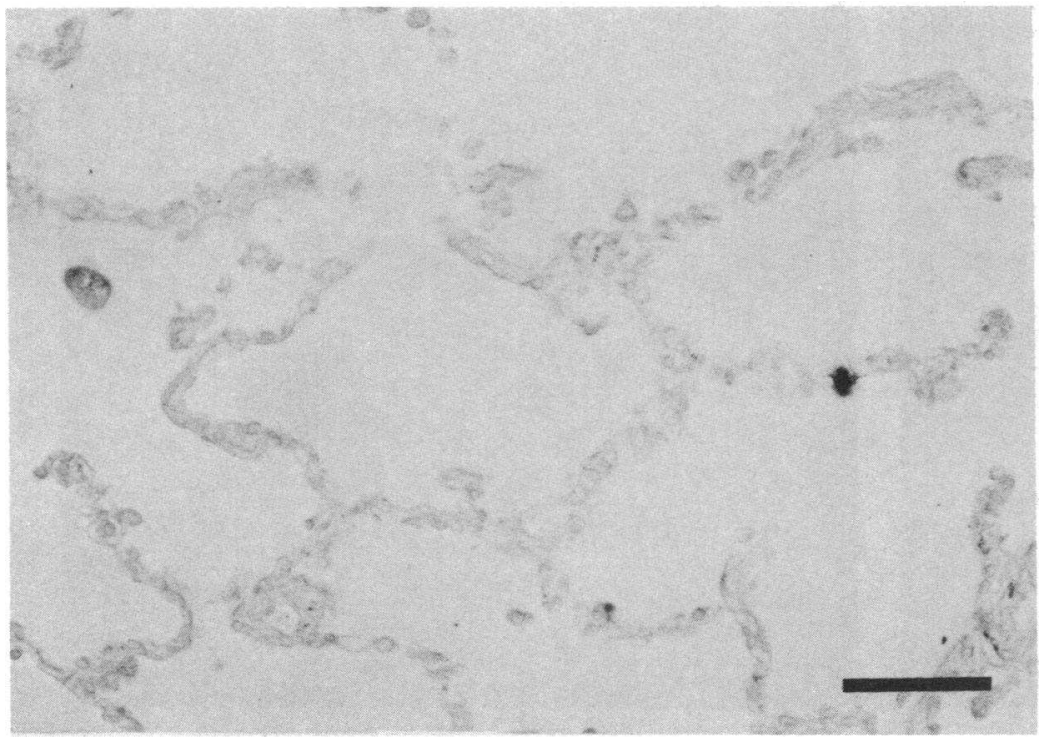

Figure 5. Normal human lung stained with Anti-pC by the avidin biotin immunoperoxidase method. At the far right an intravascular leukocyte is stained black due to its myeloperoxidase activity. The interstitial cells are unstained. Bar, $25 \mu \mathrm{m}$. the tendon sheath. After $21 \mathrm{~d}$, the tendon was removed, fixed, and processed for immunohistochemistry.

\section{Results}

\section{Characterization of procollagen I antibody}

Anti-pC is an IgG that specifically immunoprecipitated procollagen type I from fibroblast medium (Fig. 1, lanes $l$ and 2). To map the binding site of Anti-pC, we used several selective enzyme digestions of procollagen to release specific domains and isolated physiologically processed collagen precursors from IMR-90 medium. Pepsin treatment of culture medium converts type I procollagen to collagen by removing the amino and carboxyterminal propeptides as well as telopeptide extensions from procollagen. Anti-pC did not immunoprecipitate any polypeptides from pepsin-treated fibroblast culture medium. Therefore, Anti-pC bound to either the propeptide or telopeptide regions of type I procollagen (Fig. 1, lanes 3 and 4).

Digestion of type I procollagen with purified bacterial collagenase selectively degraded triple helical domains of procollagen, thus releasing intact the carboxyterminal telopeptide and disulfide crosslinked propeptide domains $(14,15)$. After digestion of labeled fibroblast medium with highly purified bacterial collagenase, several noncollagenous polypeptides remained (Fig. 1, lane 5). Immunoprecipitation of this mixture yielded two polypeptides with apparent reduced molecular masses of 35 and 32 $\mathrm{kD}$ (Fig. 1, lane 6). Unreduced, these polypeptides migrated with apparent molecular mass of $110 \mathrm{kD}$ (Fig. 1, lane 7). The apparent molecular mass of this disulfide crosslinked polypeptide is similar to previous estimates of the molecular mass of the trimeric carboxyterminal propeptides of type I procollagen $(14,15)$.

Vertebrate collagenase (purified human skin fibroblast collagenase, generously provided by Dr. Howard F. Welgus, Dermatology Division, The Jewish Hospital of St. Louis, St. Louis, MO) cleaves type I procollagen at a site in the collagenous domain approximately three quarters of the length from the amino terminus, releasing a trimeric disulfide crosslinked fragment comprised of the carboxyterminal one quarter of the collagenous domains and the intact carboxyterminal propeptides (13). Fibroblast collagenase released from labeled fibroblast medium two major pairs of polypeptides $\left(M_{\mathrm{r}}=120,150,63\right.$, and $56 \mathrm{kD}$ reduced) representing the aminoterminal and carboxyterminal mammalian collagenase cleavage products of type I procollagen, respectively (Fig. 1, lane 8 ). The smaller of these pairs representing the carboxyterminal vertebrate collagenase cleavage product was selectively immunoprecipitated with Anti-pC (Fig. 1 , lane 9). Thus, both bacterial and vertebrate collagenase digestion results were compatible with Anti-pC binding to a site either in the carboxyterminal telopeptide or propeptide domain of type I procollagen.

To further evaluate the Anti-pC epitope, we isolated intact and partially processed procollagen using DEAE-cellulose chromatography (Fig. 2) (7). Anti-pC specifically precipitated pCand intact type I procollagen but not $\mathrm{pN}$ - or fully processed collagen (Fig. 3). Because processing of procollagen by cells and tissues to pN-collagen results in cleavage between the carboxyterminal telopeptide and propeptide domains, these data suggest that Anti-pC binds to the carboxyterminal domain of type I procollagen (5).

\section{Immunostaining results}

Relationship of Anti-pC staining to the collagen-synthesizing activity of embryonic and adult tissues. As expected from previous results $(16,19)$, Anti-pC stained cultured IMR-90 matrix identically to anti-fibronectin antibodies (not shown). This staining, as well as the staining of fibrotic lung, was completely prevented by formalin fixation. Anti-pC also stained cells in embryonic chick tissues known to be actively synthesizing type I collagen, including osteoblasts and tendon fibroblasts (6) (Fig. $4 \mathrm{~A}$ ). To explore the relationship between onset of collagen synthesis induced by wounding and the acquisition of fibroblast staining by Anti-pC, we stained normal and wounded adult chicken tendon. As shown in Fig. $4 B$, unwounded chicken tendon did not stain with Anti-pC. Three weeks after wounding, when collagen synthesis was shown to be greatly increased (18), the wound site contained abundant fibroblasts with heavy intracellular staining (Fig. $4 \mathrm{C}$ ), whereas the same tendon exhibited no staining $1 \mathrm{~cm}$ distal to the wound site (not shown).

Staining of normal and fibrotic lung with Anti-pC. Although normal lung (Fig. 5) lacked detectable staining with Anti-pC, 

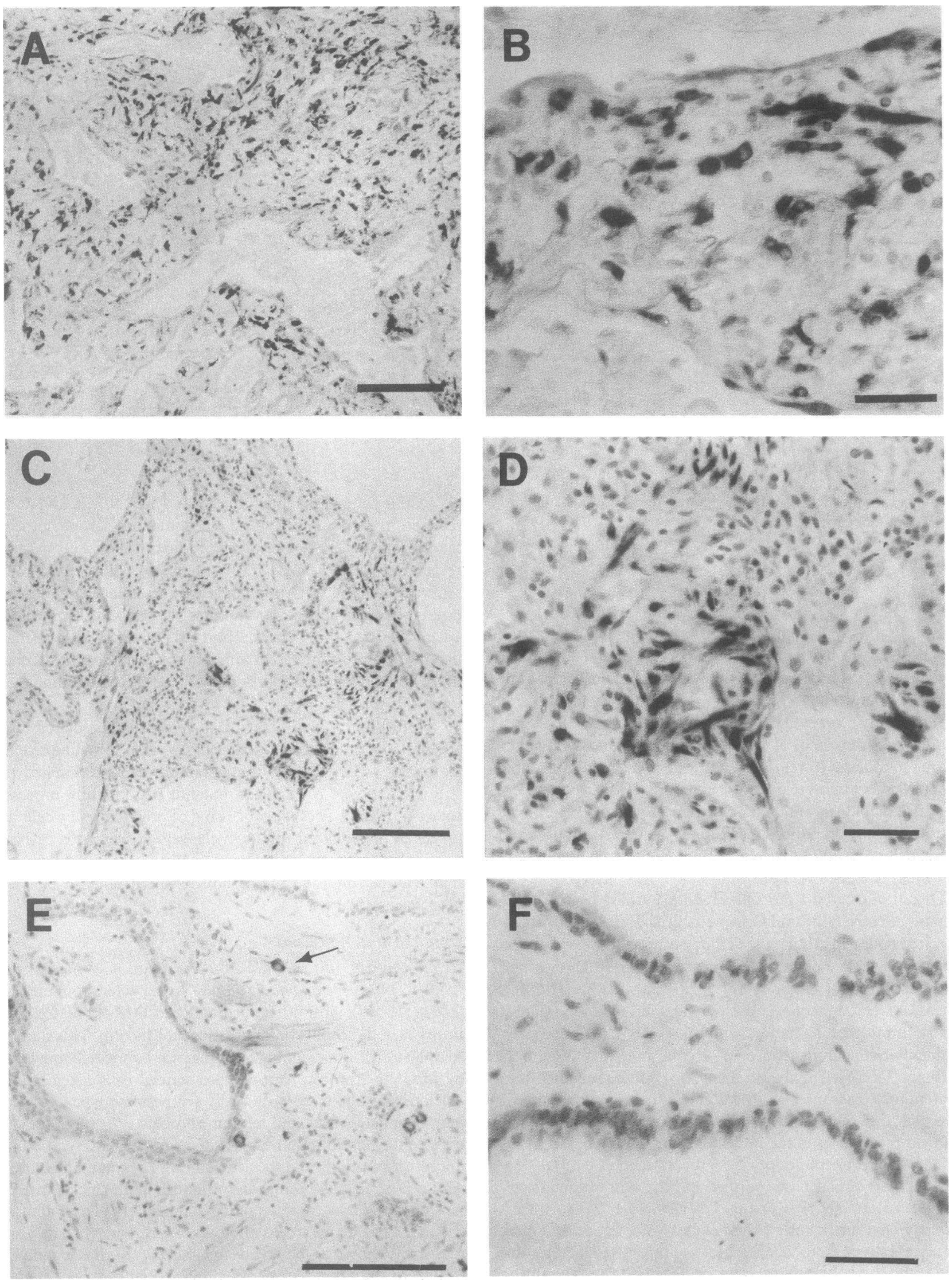

Figure 6. Spectrum of staining observed with Anti-pC in biopsies from patients with pulmonary fibrosis. $6 A$ and $B$ are from patient C.S. with rapidly progressive pulmonary fibrosis and rheumatoid disease. $A$ is a low power view showing numerous cells in the thickened alveolar septae stained black with Anti-pC immunoperoxidase stain. Bar, $50 \mu \mathrm{m} . B$ is a higher magnification of the same preparation as $A$ demonstrating the fibroblast morphology of the stained cells and the failure of Anti-pC to stain extracellular collagen. The slight darkening of the alveolar lining cells 
control experiments with antibodies to fibronectin, laminin, and type IV collagen (not shown) gave the expected localization to basal lamina (20). Thus, Anti-pC stained cultured lung fibroblast matrix, previously demonstrated to contain procollagen type I (16), as well as embryonic and healing adult tendons but not normal adult tendon or lung.

Lung biopsies from six patients with pulmonary interstitial fibrosis, either idiopathic or related to other diseases (Table I), were stained for type I procollagen with Anti-pC. The spectrum of staining patterns observed is illustrated in Fig. 6, which contains low and high power views of results obtained in three different patients. Fig. 6, $A$ and $B$ is a biopsy obtained from patient C.S. who had rheumatoid disease and accelerated fibrosis with a fatal outcome (21). Anti-pC stained myriad fibroblastic cells in the greatly thickened interstitium. A higher magnification image of this specimen shown in Fig. $6 \mathrm{~B}$ displays the fibroblastic morphology of the stained cells as well as the total absence of extracellular staining. Patient D.G., who had a more slowly progressive course, showed clusters of positively stained cells (Fig. $6 \mathrm{C}$ ), which at higher power were fibroblastic in appearance (Fig. $6 D$ ). However, Anti-pC was not a general stain for all lung fibroblasts, as results in clinically quiescent disease demonstrated. For example, patient E.M. had idiopathic pulmonary fibrosis for many years. The disease progressed very slowly and culminated in respiratory failure and death. The lung, massively fibrotic and honeycombed, contained abundant fibroblasts but no fibroblastic cells stained with Anti-pC (Fig. 6, $D$ and $E$ ).

\section{Discussion}

Immunoprecipitation of the domains of procollagen type I, obtained by selective enzymatic digestion, and of physiologically processed forms of type I procollagen are compatible with AntipC binding to the carboxyterminal domain of type I procollagen. Anti-pC did not immunoprecipitate type III procollagen from the same cultures. Although we have not performed competitive binding assays, it is unlikely that Anti-pC will crossreact with other types of procollagen. Even polyclonal antisera to the carboxyterminal domain of type I procollagen are highly selective for type I procollagen (15).

Anti-pC has the properties of an antibody specifically detecting type I collagen-synthesizing cells. Embryonic chick tissues actively synthesizing type I procollagen such as osteoblasts and tendon fibroblasts exhibit heavy intracellular staining, whereas adult tendon fibroblasts do not stain. Fibroblasts at the wound site of lacerated adult chicken tendons developed marked intracellular staining, thus demonstrating that Anti-pC stains wound fibroblasts synthesizing type I collagen. Interestingly, Anti-pC stained lung fibroblasts in biopsies from patients with clinically active pulmonary fibrosis, but not normal lung tissue. Biopsies from a larger series of patients must be studied to correlate an- tibody staining with disease activity. However, we can conclude that cells containing procollagen type I in vivo can be easily identified with Anti-pC. Moreover, in active pulmonary fibrosis, the number of fibroblasts staining for procollagen and their average intracellular collagen content (and almost certainly their synthetic activity) is increased. Thus, it appears clear from this and related studies that unknown factors in pulmonary fibrosis stimulate both increased numbers of fibroblasts and a more active fibroblast synthetic phenotype $(1,3,21,22)$. Lung fibroblasts staining with Anti-pC could contain more procollagen than fibroblasts from quiescent lung simply because procollagen secretion is somehow impeded, as in the case of ascorbate deprivation (23). This is unlikely, as dermal fibroblasts maintained in vitro under ascorbate-replete conditions, where procollagen secretion should be optimal, have intracellular pools of type I procollagen readily detectable by similar immunohistological techniques (19). Moreover, we have observed similar intracellular staining of IMR-90 cultured with ascorbate using Anti-pC.

There are no reported studies on the immunohistochemical localization of type I procollagen in human lung fibrosis, and few studies employing antibodies specific for procollagen in any human tissue. In embryonic and adult skin, immunoferritin staining shows persistence of types I and III collagen aminoterminal propeptides in collagen fibrils, whereas the carboxyterminal propeptide is not associated with collagen fibrils $(24,25)$. Antibodies to the aminoterminal propeptide of type III procollagen stained extracellular reticulin and collagen in liver (26). Notably, hepatocytes in cirrhotic liver exhibited intracellular staining similar to that seen in this study, suggesting heightened synthesis of type III collagen by hepatocytes (26). In fibrotic human lung, immunohistochemical detection of types I and III collagen has revealed apparent increases in type III that correlate somewhat with disease activity (4), but no studies of procollagen staining were performed.

Anti-pC may prove useful in the noninvasive assessment of disease activity. The absence of extracellular staining for the carboxyterminal domain of type I procollagen in chicken embryos and adult human fibrotic lung suggests that this domain is rapidly cleaved and removed in vivo, as previously suggested (5). Hence, quantitative immunoassays of serum or bronchial lavage fluid for the carboxyterminal propeptide may be useful in assessing collagen synthesis and deposition. Several investigators have used quantitative assays for type III collagen precursor extension peptides ("propeptides") to study lung and liver fibrosis. Consistent with histochemical findings cited above, the amino terminal propeptide of collagen type III is elevated in serum of patients with alcoholic liver disease and hepatic fibrosis where increased concentrations may correlate with disease activity assessed histologically or clinically $(27,28)$. Elevated bronchoalveolar lavage fluid and serum levels of type III procollagen $\mathrm{N}$-terminal peptide have also been found in patients with idio-

did not appear to be due to peroxidase reaction product. The smaller stained circular profiles are fibroblasts cut in cross section, with reaction product surrounding the nucleus. Only some cells have nuclei within the plane of the section, so the rest appear solidly stained. Some cells in the figure appear to have two holes. Whether this indicates binucleate cells or two separate adjacent cells cannot be determined. Bar, $10 \mu \mathrm{m}$. $6 C$ and $6 \mathrm{D}$ are from patient D.G. with active idiopathic pulmonary fibrosis. $C$ demonstrates that cells reactive with Anti-pC occur in focal clusters. Bar, $50 \mu \mathrm{m}$. $D$ is a higher power view to show the fusiform shape of the reactive cells. Bar, $10 \mu \mathrm{m}$. Both images are immunoperoxidase stained with hematoxylin counter staining. $6 E$ and $F$ are from patient E.M. with long standing, clinically quiescent idiopathic pulmonary fibrosis. $E$ demonstrates severe fibrosis with honeycombing. The few oval cells with positively stained cytoplasm (arrow) are mast cells. They also reacted in control sections incubated with normal mouse immunoglobulin owing to their endogenous peroxidase activity. There is no specific staining by Anti-pC. Bar, $50 \mu \mathrm{m} . F$ is a higher magnification of the airspace wall showing the absence of staining with Anti-pC. A hematoxylin counterstain has been used to bring out the nuclei of the fibroblasts. Bar, $20 \mu \mathrm{m}$. 
pathic fibrotic lung disease and sarcoidosis $(29,30)$. These peptides likely appear in plasma and bronchoalveolar lavage fluid because they are removed rapidly after collagen secretion. Because collagen type $I$ is the major collagen synthesized in lung (31), levels of carboxyterminal propeptide in biological fluids obtained from patients with pulmonary fibrosis warrant evaluation.

Finally, the carboxyterminal antigen present in fetal lung fibroblast pericellular matrix deposited in tissue culture is not present in vivo, where the antigen was only detected intracellularly. This result indicates that the posttranslational proteolytic processing of procollagen is much more complete in lung and other organs than in the cultured IMR-90 we studied or in adult dermal fibroblasts $(19,32)$. Whereas fibroblast culture is a convenient and therefore frequently used model, the fidelity with which this model reflects the complex events of collagen synthesis, processing, and deposition in vivo is uncertain.

\section{Acknowledgments}

This work was supported by National Institutes of Health grants HL36244 and HL-29594. John McDonald was an Established Investigator of the American Heart Association during this work; Mary Lou Matheke was a Research Fellow of the American Lung Association.

\section{References}

1. Kirk, J. M. E., P. E. Da Costa, M. Turner-Warwick, R. J. Littleton, and G. J. Laurent. 1986. Biochemical evidence for an increased and progressive deposition of collagen in lungs of patients with pulmonary fibrosis. Clin. Sci. (Lond.). 70:39-45.

2. Watters, L. C., T. E. King, M. I. Schwartz, J. A. Waldron, R. E. Stanford, and R. M. Cherniac. 1986. A clinical, radiographic, and physiologic assessment of patients with idiopathic pulmonary fibrosis. Am. Rev. Respir. Dis. 133:97-103.

3. Zapol, W. M., R. L. Trelstad, J. W. Coffey, I. Tsai, and R. A. Salvador. 1979. Pulmonary fibrosis in severe acute respiratory failure. Am. Rev. Respir. Dis. 119:547-554.

4. Bateman, E. D., M. Turner-Warwick, P. L. Haslam, and B. C. Adelmann-Grill. 1983. Cryptogenic fibrosing alveolitis: prediction of fibrogenic activity from immunohistochemical studies of collagen types in lung biopsy specimens. Thorax. 38:93-101.

5. Fessler, J. H., and L. I. Fessler. 1978. Biosynthesis of procollagen. Annu. Rev. Biochem. 47:129-162.

6. Kao, W. W. Y., R. A. Berg, and D. J. Prockop. 1977. Kinetics for the secretion of procollagen by freshly isolated tendon cells. J. Biol. Chem. 252:8391-8397.

7. Uitto, J., B. A. Booth, and K. L. Polak. 1980. Collagen biosynthesis by human skin fibroblasts. II. Isolation and further characterization of type I and type II procollagens synthesized in culture. Biochim. Biophys. Acta. 624:545-561.

8. Shulman, M., C. D. Wilde, and G. Kohler. 1978. A better cell line for making hybridomas secreting specific antibodies. Nature (Lond.). 276:269-270.

9. McDonald, J. A., and D. G. Kelley. 1984. Specific binding of fibronectin-antifibronectin immune complexes to procollagen: a new pitfall in immunostaining. J. Cell Biol. 98:1042-1047.

10. Engvall, E., and P. Pearlmann. 1972. Enzyme-linked immunosorbent assay, ELISA. J. Immunol. 109:129-135.

11. McDonald, J. A., T. J. Broekelmann, D. G. Kelley, and B. Villiger. 1981. Gelatin-binding domain-specific anti-human plasma fibronectin Fab' inhibits fibronectin-mediated gelatin binding but not cell spreading. J. Biol. Chem. 256:5583-5587.
12. Laemmli, U. K. 1970. Cleavage of structural proteins during the assembly of the head of bacteriophge T4. Nature (Lond.). 227:680-685.

13. Byers, P. H., E. M. Click, E. Harper, and P. Bornstein. 1975. Interchain disulfide bonds in procollagen are located in a large nontriplehelical COOH-terminal domain. Proc. Natl. Acad. Sci. USA. 72:30093013.

14. Morris, N. P., L. I. Fessler, and J. H. Fessler. 1979. Procollagen propèptide release by procollagen peptidases and bacterial collagenase. J. Biol. Chem. 254:11024-11032.

15. Goldberg, B. D., R. G. Phelps, E. Kessler, M. J. Klein, and M. B. Taubman. 1985. The carboxyl fragment released by bacterial collagenase from human type I procollagen: antibodies to the propeptide determinants. Collagen Relat. Res. 5:393-404.

16. McDonald, J. A., D. G. Kelley, and T. J. Broekelmann. 1982. Role of fibronectin in collagen deposition: Fab' to the gelatin-binding domain of fibronectin inhibits both fibronectin and collagen organization in fibroblast extracellular matrix. J. Cell Biol. 92:485-492.

17. Holund, B., P. P. Clausen, and I. Clemmensen. 1981. The influence of fixation and tissue preparation on the immunohistochemical demonstration of fibronectin in human tissue. Histochemistry. 72:291299.

18. Birdsell, D. C., E. R. Tustanoff, and W. K. Lindsay. 1966. Collagen production in regenerating tendon. Plast. Reconstr. Surg. 37:504-511.

19. Phelps, R. G., A. Martinez-Hernandez, and B. D. Goldberg. 1985. Ultrastructural immunocytochemical localization of type I procollagen in cultured human fibroblasts. Collagen Relat. Res. 5:405-414.

20. Torikata, C., B. Villiger, C. Kuhn III, and J. A. McDonald. 1985. Ultrastructural distribution of fibronectin in normal and fibrotic human lung. Lab. Invest. 52:399-408.

21. Pratt, D. S., M. I. Schwartz, J. J. May, and R. B. Dreisin. 1979. Rapidly fatal pulmonary fibrosis: the accelerated variant of interstitial pneumonitis. Thorax. 34:587-593.

22. Fulmer, J. D., R. S. Bienkowski, M. J. Cowan, S. D. Breul, K. M. Bradley, V. J. Ferrans, W. C. Roberts, and R. G. Crystal. 1980. Collagen concentration and rates of synthesis in idiopathic pulmonary fibrosis. Am. Rev. Respir. Dis. 122:289-301.

23. Schwarz, R. I. 1985. Procollagen secretion meets the minimum requirements for the rate-controlling step in the ascorbate induction of procollagen synthesis. J. Biol. Chem. 260:3045-3049.

24. Fleischmajer, R., R. Timpl, L. Tuderman, L. Raisher, M. Wiestner, J. S. Perlish, and P. N. Graves. 1981. Ultrastructural identification of extension aminopropeptides of type I and III collagens in human skin. Proc. Natl. Acad. Sci. USA. 78:7360-7364.

25. Fleischmajer, R., B. R. Olsen, R. Timpl, J. S. Perlish, and O. Lovelace. 1983. Collagen fibril formation during embryogenesis. Proc. Natl. Acad. Sci. USA. 80:3354-3358.

26. Sakakibara, K., S. Igarashi, and T. Hatahara. 1985. Localization of type III procollagen aminopeptide antigenicity in hepatocytes from cirrhotic human liver. Virchows Arch. A. Cell Pathol. 408:219-228.

27. Savolainen, E. R., B. Goldberg, M. A. Leo, M. Velez, and C. S. Lieber. 1984. Diagnostic value of serum procollagen peptide measurements in alcoholic liver disease. Alcohol. Clin. Exp. Res. 8:384-389.

28. Galambos, M. R., D. C. Collins, and J. T. Galambos. 1985. A radioimmunoassay procedure for type III procollagen: its use in the detection of hepatic fibrosis. Hepatology (Balt.). 5:38-42.

29. Low, R. B., K. R. Cutroneo, G. S. Davis, and M. S. Giancola. 1983. Lavage type III procollagen $\mathrm{N}$-terminal peptides in human pulmonary fibrosis and sarcoidosis. Lab. Invest. 48:755-759.

30. Kirk, J. M., P. L. Haslam, G. J. Laurent, and M. Turner-Warwick. 1984. Serum type III (procollagen) peptide concentration in cryptogenic fibrosing alveolitis and its clinical relevance. Thorax. 39:726-732.

31. Clark, J. G., C. Kuhn, J. A. McDonald, and R. P. Mecham. 1983. Lung connective tissue. Int. Rev. Connect. Tissue Res. 10:249-331.

32. Taubman, M. B., and B. Goldberg. 1976. The processing of procollagen in cultures of human and mouse fibroblasts. Arch. Biochem. Biophys. 173:490-494. 